\title{
Polyhedra associated with identifying codes in graphs
}

\author{
Gabriela R. Argiroffo ${ }^{\mathrm{a}, 1}$, Silvia M. Bianchi ${ }^{\mathrm{a}, 1}$, Yanina P. Lucarini ${ }^{\mathrm{b}, 1}$, Annegret K. Wagler ${ }^{\mathrm{c}, 1}$ \\ ${ }^{a}$ Universidad Nacional de Rosario (Dept. de Matemática), Rosario, Argentina \\ ${ }^{b}$ Universidad Nacional de Rosario (Dept. de Matemática), Rosario, Argentina. CONICET \\ ${ }^{c}$ Université Blaise Pascal (Clermont-Ferrand II, LIMOS UMR CNRS 6158), Aubière, France
}

\begin{abstract}
The identifying code problem is a newly emerging search problem, challenging both from a theoretical and a computational point of view, even for special graphs like bipartite graphs. Hence, a typical line of attack for this problem is to determine minimum identifying codes of special graphs or to provide bounds for their size.

In this work we study the associated polyhedra and present some general results on their combinatorial structure. We demonstrate how the polyhedral approach can be applied to find minimum identifying codes for special graphs, and discuss further lines of research in order to obtain strong lower bounds stemming from linear relaxations of the identifying code polyhedron, enhanced by suitable cutting planes to be used in a B\&C framework.
\end{abstract}

Key words: identifying code polyhedron, identifying code clutter, odd hypercycles

\section{Introduction}

Many search problems as, e.g., fault detection in networks, fire detection in buildings, or performing group tests, can be modeled by so-called identifying codes in graphs [17].

Consider a graph $G=(V, E)$ and denote by $N[i]=\{i\} \cup N(i)$ the closed neighborhood of $i$, i.e., the node $i$ together with all its neighbors. A subset $C \subseteq V$ is dominating (resp. separating) if $N[i] \cap C$ are non-empty (resp. distinct) sets for all $i \in V$. An identifying code of $G$ is a node subset which is dominating and separating, see Fig. 1 for illustration.

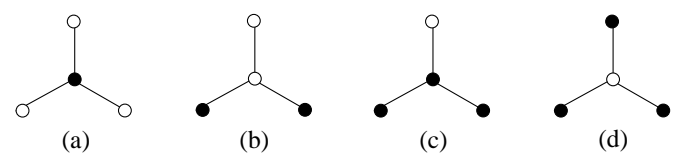

Figure 1: A graph, where the subset of black nodes forms (a) a dominating (but not separating) set, (b) a separating (but not dominating) set, and (c), (d) minimum identifying codes.

Email addresses: garua@f ceia.unr.edu.ar (Gabriela R. Argiroffo), sbianchi@f ceia.unr.edu.ar (Silvia M. Bianchi), lucarini@fceia.unr.edu.ar (Yanina P. Lucarini), wagler@isima.fr (Annegret K. Wagler)

${ }^{1}$ This work was supported by an ECOS-MINCyT cooperation France-Argentina, A12E01. 
Not every graph $G$ admits an identifying code or is identifiable: this holds if and only if there are no true twins in $G$, i.e., there is no pair of distinct nodes $i, j \in V$ with $N[i]=N[j]$ [17]. On the other hand, for every identifiable graph, its whole node set trivially forms an identifying code.

The identifying code number $\gamma^{I D}(G)$ of a graph $G$ is the minimum cardinality of and identifying code of $G$. Determining $\gamma^{I D}(G)$ is in general NP-hard [9]. From a combinatorial point of view, the problem has been actively studied during the last decade. Typical lines of attack are to determine minimum identifying codes of special graphs or to provide bounds for their size. Closed formulas for the exact value of $\gamma^{I D}(G)$ have been found so far only for restricted graph families (e.g. for paths and cycles by [8] and for stars by [15]). A linear time algorithm to determine $\gamma^{I D}(G)$ if $G$ is a tree was provided by [5], but for many other graph classes where several other in general hard problems are easy to solve, it turned out that the identifying code problem remains hard. This includes bipartite graphs [9] and two classes of chordal graphs, namely split graphs and interval graphs [11]. This motivates the study of bounds for $\gamma^{I D}(G)$. For instance, a canonical lower bound is $\lceil\log (n+1)\rceil \leq \gamma^{I D}(G)$ for general graphs $G$ of order $n$ by [17]. The trivial upper bound $\gamma^{I D}(G) \leq n$ has been improved for connected graphs $G$ to $\gamma^{I D}(G) \leq n-1$ by [15] (with stars being examples where this bound is tight) and for line graphs to $\gamma^{I D}(L(G)) \leq 2|V(G)|-5$ by [12].

As polyhedral methods have been already proved to be successful for several NP-hard combinatorial optimization problems, our aim is to apply such techniques to the identifying code problem. For that, a reformulation as set covering problem is in order. For a 0/1-matrix $M$ with $n$ columns, the set covering polyhedron is $Q^{*}(M)=\operatorname{conv}\left\{x \in \mathbf{Z}_{+}^{n}: M x \geq \mathbf{1}\right\}$ and $Q(M)=$ $\left\{x \in \mathbf{R}_{+}^{n}: M x \geq \mathbf{1}\right\}$ is its linear relaxation. A cover of $M$ is a $0 / 1$-vector $x$ such that $M x \geq \mathbf{1}$, and the covering number $\tau(M)$ equals $\min \mathbf{1}^{T} x, x \in Q^{*}(M)$ (see Section 2 for more details).

We obtain such a constraint system $M x \geq \mathbf{1}$ for the identifying code problem as follows. Domination clearly requires that any identifying code $C$ intersects the closed neighborhood $N[i]$ of each node $i \in V$. Separation means that no two intersections $C \cap N[i]$ and $C \cap N[j]$ are equal or, equivalently, that $C$ intersects each symmetric difference $N[i] \triangle N[j]$ for distinct nodes $i, j \in V$. Hence, the following constraints encode the domination and separation requirements:

$$
\begin{array}{rlll}
\min \mathbf{1}^{T} x & & \\
x(N[j])=\sum_{i \in N[j]} x_{i} & \geq 1 \forall j \in V & & \text { (domination) } \\
x(N[j] \Delta N[k])=\sum_{i \in N[j] \Delta N[k]} x_{i} & \geq 1 \forall j, k \in V, j \neq k & \text { (separation) } \\
x & \in\{0,1\}^{|V|} &
\end{array}
$$

Let $M_{I D}(G)$ be the resulting identifying code matrix of $G$, i.e., the matrix having as rows the incidence vectors of the closed neighborhoods of the nodes of $G$ and their pairwise symmetric differences. Accordingly, we define the identifying code polyhedron of $G$ as

$$
P_{I D}(G)=Q^{*}\left(M_{I D}(G)\right)=\operatorname{conv}\left\{x \in \mathbf{Z}_{+}^{|V|}: M_{I D}(G) x \geq \mathbf{1}\right\} .
$$

It is clear from the definition that a graph is identifiable if and only if none of the symmetric differences results in a zero-row of $M_{I D}(G)$, and that $\gamma^{I D}(G)$ equals the covering number $\tau\left(M_{I D}(G)\right)=\min \mathbf{1}^{T} x, x \in P_{I D}(G)$.

Our aim is to apply the polyhedral approach to find minimum identifying codes. We first provide some definitions and results related to covering polyhedra (Section 2), then we focus on general properties of the identifying code polyhedron $P_{I D}(G)$ and introduce the canonical linear relaxation (Section 3). Afterwards, we discuss several lines to apply polyhedral techniques. In Section 4, we present cases where $M_{I D}(G)$ falls into a class of matrices for which the set covering 
polyhedron is known and we, thus, immediately can obtain a complete description of $P_{I D}(G)$ and the exact value of $\gamma^{I D}(G)$. Furthermore, we present cases where a complete description of $P_{I D}(G)$ involves many and complicated facets, but where we can identify facet-defining substructures (related to minors of $M_{I D}(G)$ ) that allow us to derive the full rank inequality $x(V) \geq \tau\left(M_{I D}(G)\right)=$ $\gamma^{I D}(G)$ and, thus, the exact value of $\gamma^{I D}(G)$ (Section 5).

This demonstrates how polyhedral techniques can be applied in this context. We close with a discussion on future lines of research, including how the here obtained results can be extended to other classes of graphs.

Some of the results in this contribution appeared without proof in $[2,4]$.

\section{Properties of set covering polyhedra}

We introduce definitions and basic concepts related to set covering polyhedra and provide results which are crucial for the proofs in the subsequent sections.

\subsection{Preliminaries}

In a set $F$ of vectors in $\{0,1\}^{n}$, we say $y \in F$ is a dominating vector (of $F$ ) if there exits $x \in F$ with $x \leq y$. Alternatively, we say that $x$ is dominated by $y$. From now on, every matrix has 0,1 -entries, no zero columns and no dominating rows. Remind that a cover of a matrix $M$ is a vector $x \in\{0,1\}^{n}$ such that $M x \geq \mathbf{1}$. A cover of $M$ is minimal if it is not dominated by any other cover of $M$. The blocker of $M$ is the matrix whose rows are the minimal covers of $M$. It is known that $b(b(M))=M$ and, thus, we can refer to $Q^{*}(M)$ and $Q(b(M))$ as a blocking pair of polyhedra. Moreover, $\mathbf{a}$ is an extreme point of $Q(b(M))$ if and only if $\mathbf{a}^{T} x \geq 1$ is a facet defining inequality of $Q^{*}(M)$ (see [13]). In the sequel we will refer to this property as blocking duality.

Given a matrix $M$ and $j \in\{1, \ldots, n\}$, we introduce two matrix operations: the contraction of $j$, denoted by $M / j$, means that column $j$ is removed from $M$ as well as the resulting dominating rows and hence, corresponds to setting $x_{j}=0$ in the constraints $M x \geq \mathbf{1}$. The deletion of $j$, denoted by $M \backslash j$ means that column $j$ is removed from $M$ as well as all the rows with a 1 in column $j$ and this corresponds to setting $x_{j}=1$ in the constraints $M x \geq \mathbf{1}$. Then, given $M$ and $V_{1}, V_{2} \subset\{1, \ldots, n\}$ disjoint, we will say that $M / V_{1} \backslash V_{2}$ is a minor of $M$ and this minor does not depend on the order of operations or elements in $\{1, \ldots, n\}$. It is clear that $M$ is always a minor of itself and we will say that a minor $M / V_{1} \backslash V_{2}$ is proper if $V_{1} \cup V_{2} \neq \emptyset$. It is not hard to see that $b(M / j)=b(M) \backslash j$ and $b(M \backslash j)=b(M) / j$ for every $j \in\{1, \ldots, n\}$.

The contraction of a set $U$ of columns from $M$ is the matrix $M / U$ obtained by deleting the columns in $U$ and the deletion of a set $U$ of columns from $M$ is the matrix $M \backslash U$ obtained by deleting columns in $U$ and all the rows having at least one 1-entry in the columns in $U$.

A rank inequality associated with a minor $M^{\prime}=M \backslash U$ is

$$
\sum_{i \in M^{\prime}} x_{i} \geq \tau\left(M^{\prime}\right) .
$$

Remark 1. In [1], it is shown the following. If $(1)$ is a facet of $Q^{*}\left(M^{\prime}\right)$, then it is also a facet of $Q^{*}(M)$. In addition, if the rank constraint associated with some minor induces a facet defining inequality of $Q^{*}(M)$ then this inequality is also induced by a minor obtained by deletion. 


\subsection{Set covering polyhedra associated with q-roses}

Let $\mathcal{H}=(V, \mathcal{E})$ be a hypergraph with $\mathcal{E} \subseteq 2^{V}$ and let $M(\mathcal{H})$ denote its incidence matrix, i.e., $M(\mathcal{H})$ encodes row-wise the incidence vectors of the hyperedges in $\mathcal{E}$. Given $n \geq q \geq 2$, let $\mathcal{R}_{n}^{q}=(V, \mathcal{E})$ be the hypergraph where $V=\{1, \ldots, n\}$ and $\mathcal{E}$ contains all $q$-element subsets of $V$. Nobili and Sassano [18] called the incidence matrix of $\mathcal{R}_{n}^{q}$ the complete q-rose of order $n$ and denoted it by $R_{n}^{q}$. In [19] it is proved the following result.

Theorem 2 ([19]). For $n \geq q \geq 2$, the inequality

$$
\sum_{i=1}^{n} x_{i} \geq \tau\left(R_{n}^{q}\right)=n-q+1
$$

is a facet defining inequality for $Q^{*}\left(R_{n}^{q}\right)$.

For the sake of completeness, we here present the unpublished proofs of the results in [4] describing the set covering polyhedron of $q$-roses of order $n$. We start with the study of minors of $R_{n}^{q}$. It can be easily observed that,

Remark 3. For $n \geq q \geq 2$ and $i \in\{1, \ldots, n\}$

1. $R_{n}^{q} \backslash i=R_{n-1}^{q}$.

2. $R_{n}^{q} / i=R_{n-1}^{q-1}$.

In addition, the next result proves that the blocker of a complete $q$-rose is a complete $n-q+1$ rose.

Lemma 4. Let $n \geq q \geq 2$, then $b\left(R_{n}^{q}\right)=R_{n}^{n-q+1}$.

Proof. From Theorem 2, $\tau\left(R_{n}^{q}\right)=n-q+1$. Let $b$ a 0/1-vector with $n-q+1$ entries at value one. By definition every row of $R_{n}^{q}$ has $n-q$ entries at value zero. It is easy to check that $R_{n}^{q} b \geq \mathbf{1}$; i.e., $b$ is a minimum cover of $R_{n}^{q}$. It follows that $R_{n}^{n-q+1}$ is a row submatrix of $b\left(R_{n}^{q}\right)$. Let $b^{\prime}$ be a $0 / 1$-vector with more than $n-q+1$ entries at value one. It is clear that $b^{\prime}$ includes one of the rows in $R_{n}^{n-q+1}$. Hence $b^{\prime}$ is not a minimal cover of $R_{n}^{q}$. Then, the rows in $R_{n}^{n-q+1}$ are the only minimal covers of $R_{n}^{q}$, i.e., $b\left(R_{n}^{q}\right)=R_{n}^{n-q+1}$.

Theorem 5. Let $n \geq q \geq 2$. The point $\bar{x}$ is a fractional extreme point of $Q\left(R_{n}^{q}\right)$ if and only if

$$
\bar{x}_{i}=\left\{\begin{array}{cc}
\frac{1}{q-s} & \text { if } i \notin C_{s}, \\
0 & \text { if } i \in C_{s},
\end{array}\right.
$$

where $s \in\{0, \ldots, q-2\}$ and $C_{s} \subset\{1, \ldots, n\}$, with $\left|C_{s}\right|=s$.

Proof. Firstly consider $\bar{x}=\frac{1}{q} \mathbf{1}$, then $s=0$ and $C_{s}=\emptyset$. It is immediate that $R_{n}^{q} \bar{x}=\mathbf{1}$. In order to prove that $\bar{x}$ is an extreme point we need to find $n$ linearly independent constraints of the system $R_{n}^{q} x \geq \mathbf{1}$.

Then, for every $i=1, \ldots, q+1$, we select a row $f_{i}$ such that

$$
\left(f_{i}\right)_{j}= \begin{cases}0 & \text { if } j=n-q-1, \ldots, n \text { o } j=i \\ 1 & \text { otherwise }\end{cases}
$$

and, for every $i=q+2, \ldots, n$, we select a row $f_{i}$ of $R_{n}^{q}$ such that the last 1 -entry is at position $i$. 
The considered rows can be reordered in a matrix $M$ in the following way

$$
M=\left(\begin{array}{c|c}
E-I & 0 \\
\hline 0 & I
\end{array}\right),
$$

where $E$ is the square matrix with all its entries at value one.

The submatrix $E-I$ has rank $q+1$, since it can be easily checked that $(E-I)^{-1}=\frac{1}{n-1} E-I$. It follows that $M$ has rank $n$ and $\bar{x}$ is an extreme point of $Q\left(R_{n}^{q}\right)$.

It remains to prove that if $s=0$ then $\bar{x}=\frac{1}{q} \mathbf{1}$ is the only fractional extreme point of $Q\left(R_{n}^{q}\right)$.

Let $\bar{y}$ be a fractional extreme point of $Q\left(R_{n}^{q}\right)$ with $s=0$. Then the $n$ linearly independent facet inducing inequalities that $\bar{y}$ satisfies at equality are associated with a square row submatrix $M^{\prime}$ of $R_{n}^{q}$ with rank $n$. Observe that if $M^{\prime} x=\mathbf{1}$ has a unique solution then $\bar{y}=\bar{x}$.

Now, consider $s \in\{1, \ldots, q-1\}$ and an extreme point $\bar{x}$ defined by (2). Observe that $\bar{x} \in$ $Q\left(R_{n}^{q}\right) \cap\left\{x: x_{i}=0\right.$ for all $\left.i \in C_{s}\right\}$. From Remark 3 we have that $R_{n}^{q} / C_{s}=R_{n-s}^{q-s}$, hence $\bar{x}$ can be written as $(\bar{z}, 0)$ where $\bar{z}=\frac{1}{q-s} \mathbf{1}$ with $\bar{z} \in Q\left(R_{n-s}^{q-s}\right)$. As a consequence of the case $s=0$ already proved, $\bar{z}$ is an extreme point of $Q\left(R_{n-s}^{q-s}\right)$ and then $\bar{x}$ is an extreme point of $Q\left(R_{n}^{q}\right)$.

Conversely, let $\bar{x}$ be an extreme point of $Q\left(R_{n}^{q}\right)$ and suppose it has zero components. Let $C_{s}=\left\{i: \bar{x}_{i}=0\right\}$. Then, the point $\bar{z} \in \mathbb{R}^{n-\left|C_{s}\right|}$ such that $\bar{z}_{i}=\bar{x}_{i}, i \in\{1, \ldots, n\}-C_{s}$ is an extreme point of $Q\left(R_{n}^{q} / C_{s}\right)$. From Remark 3.(ii), if $s=\left|C_{s}\right|$ we have that $\bar{z}$ is an extreme point of $R_{n-s}^{q-s}$ with no zero components. Hence, $\bar{z}=\frac{1}{q-\left|C_{s}\right|} \mathbf{1}$. Then we have,

$$
\bar{x}_{i}=\left\{\begin{array}{cl}
\frac{1}{q-s} & \text { si } i \notin C_{s} \\
0 & \text { si } i \in C_{s} .
\end{array}\right.
$$

By blocking duality, an immediate consequence of Theorem 5 is:

Corollary 6. Let $n \geq q \geq 2$. A non-Boolean inequality $a x \geq 1$ is a facet defining inequality for $Q^{*}\left(b\left(R_{n}^{q}\right)\right)=Q^{*}\left(R_{n}^{n-q+1}\right)$ if and only if ax $\geq 1$ can be written as $x\left(A_{s}\right) \geq q-s$ for some $A_{s} \subset\{1, \ldots, n\}$ where $s \in\{0, \ldots, q-2\}$ and $\left|A_{s}\right|=n-s$.

\section{General properties of identifying code polyhedra}

In this section, we examine general properties of identifying code polyhedra concerning their dimension and the question which of the constraints defining the canonical linear relaxation define facets. From the set covering formulation, it is clear that the inequalities

$$
\begin{aligned}
x_{i} \geq 0 & & \text { for } i \in V, \\
x(N[i]) \geq 1 & & \text { for } i \in V, \\
x(N[i] \Delta N[j]) \geq 1 & & \text { for } i, j \in V, j \neq k
\end{aligned}
$$

are always valid for $P_{I D}(G)$. The inequalities (4) are called trivial, we refer to the inequalities (5) as closed neighborhood inequalities and to the inequalities (6) as symmetric difference inequalities.

Accordingly, the identifying code matrix is composed by

$$
M_{I D}(G)=\left(\begin{array}{c}
N[G] \\
\Delta[G]
\end{array}\right),
$$


encoding row-wise the closed neighborhoods of the nodes of $G$ (in $N[G]$ ) and their pairwise symmetric differences (in $\triangle[G]$ ).

A graph $G$ is identifiable if and only if $P_{I D}(G)$ is non-empty. As $N[G]$ has no zero-row, $G$ is identifiable if and only if $\Delta[G]$ has no zero-row (i.e. if and only if $G$ has no true twins [17]).

We first address the question when $P_{I D}(G)$ is full-dimensional. It is known from Balas and $\mathrm{Ng}$ [6] that a polyhedron $Q^{*}(M)$ is full-dimensional if and only if the matrix $M$ has at least two ones per row. For $P_{I D}(G)$, this means that $G$ must not have isolated nodes (to ensure $|N[i]| \geq 2$ for all $i \in V(G))$ and that there are no two adjacent nodes $i$ and $j$ with $N[i]=N[j] \cup\{k\}$ for some node $k$ (to ensure $|N[i] \triangle N[j]| \geq 2$ for all distinct $i, j \in V(G)$ ).

Let $V_{1}(G)$ be the set of nodes $k \in V(G)$ such that $\{k\}=N[i] \Delta N[j]$ for two different nodes $i$ and $j$ in $V(G)$. We immediately obtain:

Corollary 7. Let $G$ be a graph without isolated nodes. Then, we have:

1. $P_{I D}(G)$ is full-dimensional if and only if $V_{1}(G)=\emptyset$.

2. The constraint $x_{i} \geq 0$ defines a facet of $P_{I D}(G)$ if and only if $i \notin V_{1}(G)$.

In addition, $M_{I D}(G)$ may contain rows which are equal to or dominated by other rows in $M_{I D}(G)$. We, therefore, define the corresponding clutter matrix, the identifying code clutter matrix $C_{I D}(G)$ of a graph $G$, obtained by removing repeated or dominated rows from $M_{I D}(G)$. We clearly have $P_{I D}(G)=\operatorname{conv}\left\{x \in \mathbf{Z}_{+}^{|V|}: C_{I D}(G) x \geq \mathbf{1}\right\}$. Moreover, we obtain due to Balas and $\mathrm{Ng}[6]$ :

Corollary 8. All constraints from $C_{I D}(G) x \geq \mathbf{1}$ define facets of $P_{I D}(G)$.

We obtain a linear relaxation, the fractional identifying code polyhedron $Q_{I D}(G)$ of $G$, by considering all vectors satisfying the above inequalities:

$$
Q_{I D}(G)=\left\{x \in \mathbf{R}_{+}^{|V|}: C_{I D}(G) x \geq \mathbf{1}\right\} .
$$

We, therefore, propose to firstly determine the identifying code clutter matrix $C_{I D}(G)$ and then to study which further constraints have to be added to $Q_{I D}(G)$ to obtain $P_{I D}(G)$.

In order to discuss which rows from $M_{I D}(G)$ remain in $C_{I D}(G)$ it is convenient to consider the hypergraph associated with $C_{I D}(G)$. We define the identifying code hypergraph $H_{I D}(G)$ to be the hypergraph whose incidence matrix $M\left(H_{I D}(G)\right)$ equals $C_{I D}(G)$. Clearly, $H_{I D}(G)$ cannot contain any closed neighborhood or symmetric difference containing a node from $V_{1}(G)$. In addition, we observe that if $i$ and $j$ are neither adjacent nor have a common neighbor, then $N[i]$ and $N[j]$ are disjoint, hence $N[i] \Delta N[j]=N[i] \cup N[j]$ follows and is dominated from both $N[i]$ and $N[j]$. This implies: A symmetric difference $N[i] \triangle N[j]$ is a hyperedge of $H_{I D}(G)$ only if $i$ and $j$ are adjacent or have a common neighbor.

\section{Identifying code polyhedra of complete $p$-partite graphs}

In this section, we consider complete $p$-partite graphs and establish a connection to complete 2-roses of order $n, R_{n}^{2}$, already mentioned in Section 2.2.

First we consider complete bipartite graphs $K_{m, n}$ with bipartition $A=\{1, \ldots, m\}$ and $B=$ $\{m+1, \ldots, m+n\}$. We begin with the case of stars $K_{1, n}$, i.e., $A=\{1\}$ and $n \geq 3$.

Lemma 9. For a star $K_{1, n}$, we have $H_{I D}\left(K_{1, n}\right)=K_{1+n}$ and $C_{I D}\left(K_{1, n}\right)=R_{n+1}^{2}$. 
Proof. For a star $K_{1, n}$, we have that

- $N[1]=\{1\} \cup B$,

- $N[i]=\{1, i\}$ for all $i \in B$,

- $N[1] \triangle N[i]=B-\{i\}$ for all $i \in B$,

- $N[j] \triangle N[k]=\{j, k\}$ for distinct $j, k \in B$.

This shows that $V_{1}\left(K_{1, n}\right)=\emptyset$. After removing dominated sets, namely $N[1]=\{1\} \cup B$ and $N[1] \triangle N[i]=B-\{i\}$ for all $i \in B$, we obtain that $H_{I D}\left(K_{1, n}\right)$ exactly contains all 2-element subsets of $A \cup B$ and, thus, it induces a clique $K_{1+n}$ and $C_{I D}\left(K_{1, n}\right)=R_{n+1}^{2}$ follows.

Then we deduce from Corollary 6:

Corollary 10. $P_{I D}\left(K_{1, n}\right)$ is described by the inequalities $x(C) \geq|C|-1$ for all nonempty subsets $C \subset\{1, \ldots, n+1\}$.

The above inequalities yield, for $|C|=1$, the trivial inequalities $x_{i} \geq 0$ and, for $|C|=2$, the closed neighborhood and symmetric difference inequalities $x_{i}+x_{j} \geq 1$ with $i \neq j$ describing $Q_{I D}\left(K_{1, n}\right)$. On the other hand, $C=V$ yields the full rank facet which immediately implies $\gamma^{I D}\left(K_{1, n}\right)=|V|-1$ (and provides an alternative proof for the result given in [15]).

For general complete bipartite graphs $K_{m, n}$ with $m \geq 2, n \geq 3$, we obtain:

Lemma 11. For a complete bipartite graph $K_{m, n}$, we have $H_{I D}\left(K_{m, n}\right)=K_{m}+K_{n}$ and

$$
C_{I D}\left(K_{m, n}\right)=\left(\begin{array}{cc}
R_{m}^{2} & 0 \\
0 & R_{n}^{2}
\end{array}\right) .
$$

Proof. Observe that for all $i \in A, N[i]=\{i\} \cup B$ and $N[i] \triangle N[j]=(A \cup B)-\{i, j\}$ for $j \in B$ include $N[k] \Delta N[l]=\{k, l\}$ for distinct $k, l \in B$. Symmetric considerations for $i \in B$ show that only symmetric differences $N[i] \triangle N[j]$ remain where $i, j$ come either both from $A$ or both from $B$. Thus, $H_{I D}\left(K_{1, n}\right)$ exactly contains all 2-element subsets of $A$ and all 2-element subsets of $B$.

It is known that the set of facet-defining inequalities of $Q^{*}(M)$ when $M$ is a block matrix of the form

$$
M=\left(\begin{array}{cc}
M_{1} & \mathbf{0} \\
\mathbf{0} & M_{2}
\end{array}\right)
$$

is the union of the sets of facet-defining inequalities for $Q^{*}\left(M_{1}\right)$ and $Q^{*}\left(M_{2}\right)$.

As consequence of Lemma 11, Corollary 6 and the above remark we conclude the following:

Corollary 12. $P_{I D}\left(K_{m, n}\right)$ is given by the inequalities

1. $x(C) \geq|C|-1$ for all nonempty $C \subseteq A$,

2. $x(C) \geq|C|-1$ for all nonempty $C \subseteq B$.

Moreover, $\gamma^{I D}\left(K_{m, n}\right)=|V|-2$.

This result can be further generalized for complete $p$-partite graphs $K_{n_{1}, \ldots, n_{p}}=\left(V_{1}, \ldots, V_{p}, E\right)$ where each $V_{i}=\left\{v_{i 1}, \ldots, v_{i n_{i}}\right\}$ induces a stable set and all edges between any $V_{i}$ and $V_{j}$ are present. We use $\left|V_{i}\right|=n_{i}$ for $i=1, \ldots, p,|V|=n$ and assume $n_{1} \leq n_{2} \leq \ldots \leq n_{p}$ as well as $p \geq 3$.

Firstly note that $K_{n_{1}, \ldots, n_{p}}$ is not identifiable if $n_{2}=1$ (because in this case, $V_{1}=\left\{v_{11}\right\}$ and $V_{2}=\left\{v_{21}\right\}$ holds such that $v_{11}$ and $v_{21}$ become true twins). 
Lemma 13. For a complete p-partite graph $K_{n_{1}, n_{2}, \ldots, n_{p}}$ with $n_{1}=1, n_{i}=2$ for all $i=2 \ldots, r$, and $n_{i} \geq 3$ for $i=r+1, \ldots, p$, we have:

$$
C_{I D}\left(K_{1, n_{2}, \ldots, n_{p}}\right)=\left(\begin{array}{ccccc}
0 & I_{2(r-1)} & 0 & \ldots & 0 \\
0 & 0 & R_{n_{r+1}}^{2} & & \\
\vdots & & \ddots & \ddots & \vdots \\
0 & \ldots & & 0 & R_{n_{p}}^{2}
\end{array}\right)
$$

Proof. For a complete $p$-partite graph $G=K_{n_{1}, n_{2}, \ldots, n_{p}}$ with $n_{1}=1, n_{i}=2$ for all $i=1, \ldots, r$, and $n_{i} \geq 3$ for $i=r+1, \ldots, p$, we have the following closed neighborhoods:

- $N\left[v_{11}\right]=V$,

- $N\left[v_{i 1}\right]=V-\left\{v_{i 2}\right\}$ and $N\left[v_{i 2}\right]=V-\left\{v_{i 1}\right\}$ for $i=2, \ldots, r$,

- $N\left[v_{i j}\right]=\left(V-V_{i}\right) \cup\left\{v_{i j}\right\}$ for $i=r+1, \ldots, p$

Hence, $N\left[v_{11}\right] \Delta N\left[v_{i 1}\right]=\left\{v_{i 2}\right\}$ and $N\left[v_{11}\right] \Delta N\left[v_{i 2}\right]=\left\{v_{i 1}\right\}$ for all $i=2, \ldots, r$ shows that $V_{2} \cup$ $\ldots \cup V_{r} \subseteq V_{1}(G)$. All closed neighborhoods contain at least one node from $V_{1}(G)$ and are, thus, all dominated. Moreover, all symmetric differences different from $N\left[v_{i j}\right] \Delta N\left[v_{i k}\right]=\left\{v_{i j}, v_{i k}\right\}$ for $i=r+1, \ldots, p$ are dominated:

- $N\left[v_{11}\right] \Delta N\left[v_{i j}\right]=V_{i}-\left\{v_{i j}\right\}$ for $i=r+1, \ldots, p$ is dominated by $N\left[v_{i j}\right] \Delta N\left[v_{i k}\right]\left(\right.$ by $\left.n_{i} \geq 3\right)$,

- $N\left[v_{i 1}\right] \Delta N\left[v_{i 2}\right]$ and $N\left[v_{i l}\right] \Delta N\left[v_{j k}\right]$ for all $i, j=2, \ldots, r$, as well as $N\left[v_{i l}\right] \Delta N\left[v_{j k}\right]$ for $i=2, \ldots, r, j=r+1, \ldots, p$ intersect $V_{1}(G)$.

Thus, there is no hyperedge in $H_{I D}(G)$ containing $v_{11}$. The nodes from $V_{2} \cup \ldots \cup V_{r}$ form $V_{1}(G)$ (leading to an identity matrix in $C_{I D}(G)$ ), and each $V_{i}$ with $i=r+1, \ldots, p$ induces a 2-rose of order $n_{i}$ in $C_{I D}(G)$.

As consequence of Lemma 13, Corollary 6 and the above remark on block matrices, we conclude:

Corollary 14. For a complete p-partite graph $G=K_{n_{1}, n_{2}, \ldots, n_{p}}$ with $n_{1}=1, n_{i}=2$ for all $i=2, \ldots, r$, and $n_{i} \geq 3$ for $i=r+1, \ldots, p, P_{I D}(G)$ is given by the inequalities

1. $x\left(v_{11}\right) \geq 0$ and $x\left(v_{i j}\right) \geq 0$ for all $v_{i j} \in V_{i}, i=r+1, \ldots, p$,

2. $x\left(v_{i j}\right) \geq 1$ for all $v_{i j} \in V_{i}, i=2, \ldots, r$,

3. $x\left(V^{\prime}\right) \geq\left|V^{\prime}\right|-1$ for all nonempty subsets $V^{\prime} \subseteq V_{i}$ for $i=r+1, \ldots, p$.

Moreover $\gamma^{I D}(G)=n-p+r-1$.

Using similar arguments as in the proof of Lemma 13, we obtain:

Lemma 15. For a complete p-partite graph $K_{n_{1}, n_{2}, \ldots, n_{p}}$ with $n_{i}=2$ for $i=1, \ldots, r$ and $n_{i} \geq 3$ for $i=r+1, \ldots, p$, we have:

$$
C_{I D}\left(K_{1, n_{2}, \ldots, n_{p}}\right)=\left(\begin{array}{cccc}
R_{2 r}^{2} & 0 & \ldots & 0 \\
0 & R_{n_{r+1}}^{2} & 0 & \\
\vdots & & \ddots & \vdots \\
0 & \ldots & 0 & R_{n_{p}}^{2}
\end{array}\right)
$$

Lemma 15, Corollary 6 and the above remark on block matrices imply: 
Corollary 16. For a complete p-partite graph $G=K_{n_{1}, n_{2}, \ldots, n_{p}}$ with $n_{i}=2$ for all $i=1, \ldots, r$, and $n_{i} \geq 3$ for $i=r+1, \ldots, p, P_{I D}(G)$ is given by the inequalities

1. $x(v) \geq 0$ for all $v \in V$,

2. $x\left(V^{\prime}\right) \geq\left|V^{\prime}\right|-1$ for all nonempty subsets $V^{\prime} \subseteq V_{1} \cup \ldots \cup V_{r}$,

3. $x\left(V^{\prime}\right) \geq\left|V^{\prime}\right|-1$ for all nonempty subsets $V^{\prime} \subseteq V_{i}$ for $i=r+1, \ldots, p$.

Moreover $\gamma^{I D}(G)=n-p+r-1$.

Remark 17. Note that any 2-rose minor in $C_{I D}(G)$ corresponds to a clique in $H_{I D}(G)$. Lemma 9 shows that the identifying code hypergraph of stars is a clique, and Lemma 10 implies that cliques form facet-defining substructers. In particular, every set of pairwise false twins in a graph gives rise to a clique in $H_{I D}(G)$ since for non-adjacent nodes $i$ and $j$ with $N(i)=N(j)$, we have $N[i] \Delta N[j]=\{i, j\}$ (see complete multi-partite graphs for examples). Hence, each set $V^{\prime}$ of pairwise false twins in a graph $G$ leads to a facet $x\left(V^{\prime}\right) \geq\left|V^{\prime}\right|-1$ of $P_{I D}(G)$.

\section{Identifying code polyhedra of suns}

In this section, we discuss hypercycles as further relevant substructers in $H_{I D}(G)$ that can lead to valid or facet-defining inequalities of $P_{I D}(G)$.

Let $\mathcal{H}=(V, \mathcal{E})$ be a hypergraph with $\mathcal{E} \subseteq 2^{V}$. A hypercycle $C=\left(V^{\prime}, \mathcal{E}^{\prime}\right)$ of length $m$ is a hypergraph defined by an alternating sequence $i_{1} E_{1} i_{2} \ldots i_{m} E_{m} i_{1}$ of $m$ nodes and $m$ hyperedges with $\left\{i_{j}, i_{j+1}\right\} \in E_{i}, i_{m+1}=i_{1}$. It is an induced hypergraph of $\mathcal{H}$ if $M(C)$ is a deletion minor of $M(\mathcal{H})$, i.e., if it is obtained by removing the columns outside $V^{\prime}$ and the rows with a 1-entry outside $V^{\prime}$. The result in Remark1 can be restated as follows:

Lemma 18. Let $\mathcal{H}^{\prime}=\left(V^{\prime}, \mathcal{E}^{\prime}\right)$ be an induced hypergraph of $\mathcal{H}=(V, \mathcal{E})$. The inequality $x\left(V^{\prime}\right) \geq$ $\tau\left(M\left(\mathcal{H}^{\prime}\right)\right)$ is always valid for $Q^{*}(M(\mathcal{H}))$. Moreover if it is a facet of $Q^{*}\left(M\left(\mathcal{H}^{\prime}\right)\right)$ then it is also a facet of $Q^{*}(M(\mathcal{H}))$.

In the sequel, we consider three families of suns and study hypercycles in their identifying code hypergraphs in order to determine minimum identifying codes.

It will turn out that the corresponding identifying code clutters are related to different circulant matrices. A circulant matrix is a square matrix where each row vector is shifted one element to the right relative to the preceding row. We denote by $C_{n}^{k}$ a matrix in $\{0,1\}^{n \times n}$ having as first row the vector starting with $k 1$-entries and having 0 -entries otherwise. In contrary to the case of $q$-roses, the covering polyhedron of general circulant matrices has not yet been described, even not for all matrices $C_{n}^{k}$ but only for some special cases (see [1] and [10] for further references).

A sun is a graph $G=(C \cup S, E)$ whose node set can be partitioned into $S$ and $C$, where $S=\left\{s_{1}, \ldots, s_{n}\right\}$ is a stable set and $C=\left\{c_{1}, \ldots, c_{n}\right\}$ is a (not necessarily chordless) cycle.

Here, we focus our consideration on three cases:

- $n$-suns $M_{n}$ where $C$ induces a hole and $s_{i}$ is adjacent to exactly $c_{i}$ and $c_{i+1}$ for all $1 \leq i \leq n$,

- complete suns $S_{n}$ where $C$ induces a clique and $s_{i}$ is adjacent to exactly $c_{i}$ and $c_{i+1}$ for all $1 \leq i \leq n$ and

- co-suns $\bar{S}_{n}$ (the complements of complete suns $S_{n}$ )

(indices are taken modulo $n$ ), see Figure 2 for examples. By definition, we immediately see that all such suns with $n \geq 3$ are identifiable.

We start our considerations with $n$-suns. Note that $\gamma_{I D}\left(M_{3}\right)=3$ is easy to see. 


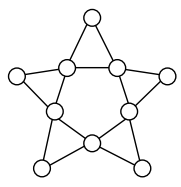

(a)

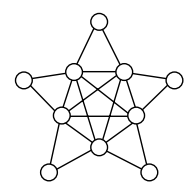

(b)

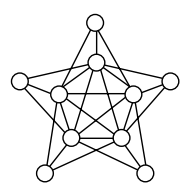

(c)

Figure 2: Three examples of suns (a) the 5 -sun $M_{5}$, (b) the complete sun $S_{5}$ and (c) its complement, the co-sun $\bar{S}_{5}$.

Theorem 19. For an $n$-sun $M_{n}=(C \cup S, E)$ with $n \geq 4$, we have

$$
C_{I D}\left(M_{n}\right)=\left(\begin{array}{cc}
I & I \\
C_{w} & I
\end{array}\right) .
$$

where $C_{w}$ is the circulant matrix whose first row is $(0,1,0, \ldots, 0)$. Moreover, $H_{I D}\left(M_{n}\right)=C_{2 n}$ and $C_{I D}\left(M_{n}\right)=C_{2 n}^{2}$.

Proof. The neighborhood matrix of $M_{n}$ can be writen as

$$
N\left[M_{n}\right]=\left(\begin{array}{cc}
C_{n}^{2} & I \\
C_{n}^{3} & C_{n}^{2}
\end{array}\right)
$$

because we have that $N\left[s_{i}\right]=\left\{s_{i}, c_{i}, c_{i+1}\right\}$ and $N\left[c_{i}\right]=\left\{c_{i-1}, c_{i}, c_{i+1}, s_{i-1}, s_{i}\right\}$. Clearly, $N\left[c_{i}\right]$ is dominated by $N\left[s_{i}\right]$ for all $i \leq n$. To find $\Delta\left[M_{n}\right]$, we consider the following cases:

- We have $N\left[s_{i}\right] \triangle N\left[c_{i}\right]=\left\{s_{i}, c_{i}, c_{i+1}\right\} \Delta\left\{c_{i-1}, c_{i}, c_{i+1}, s_{i-1}, s_{i}\right\}=\left\{c_{i-1}, s_{i-1}\right\}$ and $N\left[s_{i-1}\right] \Delta N\left[c_{i}\right]=$ $\left\{c_{i+1}, s_{i}\right\}$, which clearly dominate all rows in $N\left[M_{n}\right]$.

- Consider $c_{i}, c_{j} \in C$. If $c_{i}$ and $c_{j}$ are adjacent nodes, say, if $j=i+1$ holds, then $N\left[c_{i}\right] \Delta$ $N\left[c_{i+1}\right]=\left\{c_{i-1}, c_{i}, c_{i+1}, s_{i-1}, s_{i}\right\} \triangle\left\{c_{i}, c_{i+1}, c_{i+2}, s_{i}, s_{i+1}\right\}=\left\{c_{i-1}, c_{i+2}, s_{i-1}, s_{i+1}\right\}$ follows. Due to $\left\{c_{i-1}, s_{i-1}\right\} \subset N\left[c_{i}\right] \Delta N\left[c_{i+1}\right]$, it is dominated by $N\left[s_{i}\right] \Delta N\left[c_{i}\right]$. Otherwise, $N\left[c_{i}\right] \Delta N\left[c_{j}\right]$ clearly contains $\left\{s_{i}, c_{i}\right\}$ and is, thus, dominated by $N\left[s_{i+1}\right] \triangle N\left[c_{i+1}\right]$.

- Let $s_{i}, s_{j} \in S$. If $s_{i}$ and $s_{j}$ have a common neighbor, say, $c_{i+1}$ and $j=i+1$ holds, then $N\left[s_{i}\right] \triangle N\left[s_{i+1}\right]=\left\{s_{i}, c_{i}, c_{i+1}\right\} \Delta\left\{s_{i+1}, c_{i+1}, c_{i+2}\right\}=\left\{s_{i}, s_{i+1}, c_{i}, c_{i+2}\right\}$. Due to $\left\{s_{i}, c_{i}\right\} \subset$ $N\left[s_{i}\right] \triangle N\left[s_{i+1}\right]$, it is dominated by $N\left[s_{i+1}\right] \Delta N\left[c_{i+1}\right]$. Otherwise, $N\left[s_{i}\right]$ and $N\left[s_{j}\right]$ are disjoint and $\left\{s_{i}, c_{i}\right\} \subset N\left[s_{i}\right] \triangle N\left[s_{j}\right]$ follows, too.

Since all rows of $C_{I D}\left(M_{n}\right)$ have exactly two 1-entries, it is clear that $H_{I D}\left(M_{n}\right)$ is a graph. It is a cycle since $N\left[s_{i}\right] \Delta N\left[c_{i}\right]=\left\{c_{i-1}, s_{i-1}\right\}$ and $N\left[s_{i-2}\right] \Delta N\left[c_{i-1}\right]=\left\{c_{i}, s_{i-1}\right\}$ share the node $s_{i-1}$, and $N\left[s_{i-2}\right] \triangle N\left[c_{i-1}\right]$ and $N\left[s_{i+1}\right] \triangle N\left[c_{i+1}\right]=\left\{s_{i}, c_{i}\right\}$ share node $c_{i}$. Accordingly, its incidence matrix $C_{I D}\left(M_{n}\right)$ can be re-arranged as $C_{2 n}^{2}$ (by ordering the columns as $c_{1}, s_{1}, c_{2}, s_{2}, \ldots, c_{n}, s_{n}$ and the rows as $N\left[c_{2}\right] \triangle N\left[s_{2}\right], N\left[c_{1}\right] \triangle N\left[s_{n}\right], N\left[c_{3}\right] \triangle N\left[s_{3}\right], N\left[c_{2}\right] \triangle N\left[s_{1}\right], \ldots, N\left[c_{1}\right] \triangle N\left[s_{1}\right], N\left[c_{n}\right] \triangle$ $\left.N\left[s_{n-1}\right]\right)$.

Hence, $H_{I D}\left(M_{n}\right)$ is an even (hyper)cycle and $\tau\left(M_{n}\right)=n$ clearly holds. In addition, $C_{2 n}^{2}$ is one of the few circulant matrices where $Q^{*}\left(C_{2 n}^{2}\right)$ is known [10], and we conclude:

Corollary 20. For $M_{n}=(C \cup S, E)$ with $n \geq 4, P_{I D}\left(M_{n}\right)$ coincides with its linear relaxation $Q\left(C_{I D}\left(M_{n}\right)\right)$ and $\gamma_{I D}\left(M_{n}\right)=n$. 
Let us now consider a complete sun $S_{n}=(C \cup S, E)$ with $n \geq 4$. In contrary to $n$-suns, the identifying code clutters of complete suns have a much more complex structure [3], involving different combinations of circulant matrices, where some submatrices occur for all $n \geq 4$, others not (depending on the parity of $n$ and the size of the graph). Accordingly, the description of $P_{I D}\left(S_{n}\right)$ requires many and complex facets. However, an analysis of $C_{I D}\left(S_{n}\right)$ shows that

$S$ is an identifying code and $\gamma^{I D}\left(S_{n}\right) \leq|S|=n$. In [3] we conjectured that this bound is tight. In order to prove the conjecture, we rely on the following result:

Lemma 21. Let $S_{n}=(C \cup S, E)$ be a complete sun whith $n \geq 4$. The hyperedges $N\left[s_{i}\right], N\left[s_{i+1}\right]$, and $N\left[s_{i}\right] \Delta N\left[s_{i+1}\right]$ form a hypercycle in $H_{I D}\left(S_{n}\right)$ that induces a rank facet $x\left(\left\{c_{i}, c_{i+1}, c_{i+2}, s_{i}, s_{i+1}\right\}\right)$ $\geq 2$ of $P_{I D}\left(S_{n}\right)$.

Proof. Consider the following hyperedges from $H_{I D}\left(S_{n}\right)$ : the neighborhoods $N\left[s_{i}\right]=\left\{c_{i}, c_{i+1}, s_{i}\right\}$, $N\left[s_{i+1}\right]=\left\{c_{i+1}, c_{i+2}, s_{i+1}\right\}$ and their symmetric difference $N\left[s_{i}\right] \Delta N\left[s_{i+1}\right]=\left\{c_{i}, c_{i+2}, s_{i}, s_{i+1}\right\}$.

They form, for all $n \geq 4$, a hypercycle of length 3 with support $\left\{c_{i}, c_{i+1}, c_{i+2}, s_{i}, s_{i+1}\right\}$. It is clear that this hypercycle is obtained by deletion of the columns in $V-\left\{c_{i}, c_{i+1}, c_{i+2}, s_{i}, s_{i+1}\right\}$ in $C_{I D}\left(S_{n}\right)$. As $x\left(\left\{c_{i}, c_{i+1}, c_{i+2}, s_{i}, s_{i+1}\right\}\right) \geq 2$ for $n \geq 4$ is a facet of this deletion minor, it is also a facet of $P_{I D}\left(S_{n}\right)$ by Lemma 18 .

Theorem 22. For a complete sun $S_{n}=(C \cup S, E)$ with $n \geq 4$, the stable set $S$ is a minimum identifying code and, thus, $\gamma^{I D}\left(S_{n}\right)=n$.

Proof. Let us firstly observe that the stable set $S$ is an identifying code in $S_{n}$ : indeed, $N\left[s_{i}\right] \cap S=$ $\left\{s_{i}\right\}$ and $N\left[c_{i}\right] \cap S=\left\{s_{i-1}, s_{i}\right\}$ holds for $i=1, \ldots, n$, thus each node is dominated and separated.

In order to show that $S$ is a minimum identifying code in $S_{n}$, we consider an arbitrary identifying code $I$ in $S_{n}$ with $I \neq S$ and show that $|I| \geq n$.

Observe that $I$ contains nodes from both $C$ and $S$ : we can neither have $I \subset S$ (otherwise $I$ does not dominate any node in $S-I$ nor we can have $I \subset C$ (otherwise $I$ does not separate any two nodes in $C$ ).

In order to show $|I| \geq n$, we provide arguments implying $|I \cap C| \geq|S-I|$. Note that $S-I$ cannot contain 3 consecutive nodes $s_{i-1}, s_{i}, s_{i+1}$ from $S$ (otherwise $I \cap N\left[c_{i}\right]=I \cap C=I \cap N\left[c_{i+1}\right]$ holds). Hence, $S-I$ can be partitioned into blocks containing either a single node or two consecutive nodes from $S$, where no two blocks are consecutive. Suppose that $S-I$ consists of $p$ blocks $A_{j}$ of cardinality 1 and $q$ blocks $B_{k}$ with two consecutive nodes each and consequently $p+2 q=|S-I|$.

For each block $A_{j}=\left\{s_{i}\right\}$, it clearly follows $\left|I \cap\left\{c_{i+1}, c_{i}\right\}\right| \geq 1$ from the inequality $x\left(\left\{s_{i}, c_{i+1}, c_{i}\right\}\right)$ $\geq 1$ associated to the hyperedge $N\left[s_{i}\right]$ in $H_{I D}\left(S_{n}\right)$.

For each block $B_{k}=\left\{s_{i}, s_{i+1}\right\}$, we have by Lemma 21 that $N\left[s_{i}\right], N\left[s_{i+1}\right]$, and $N\left[s_{i}\right] \Delta$ $N\left[s_{i+1}\right]$ form a hypercycle with rank facet $x\left(\left\{c_{i}, c_{i+1}, c_{i+2}, s_{i}, s_{i+1}\right\}\right) \geq 2$, which clearly implies $\left|I \cap\left\{c_{i}, c_{i+1}, c_{i+2}\right\}\right| \geq 2$.

In addition, if $s_{i}$ and $s_{j}$ belong to different blocks of $S-I$, then the sets of their neighbors in $C$ are disjoint. This finally shows $|I \cap C| \geq p+2 q$ and implies $|I| \geq n$.

Finally, let us consider co-suns. Note that $\bar{S}_{3}=M_{3}$ and $\bar{S}_{4}=S_{4}$ holds.

Also the identifying code clutters of complete suns have a complex structure [3], involving different combinations of circulant matrices, where some submatrices occur for all $n \geq 4$, others not (depending on the parity of $n$ and the size of the graph). Accordingly, the description of $P_{I D}\left(\bar{S}_{n}\right)$ requires many and complex facets. However, an analysis of $C_{I D}\left(\bar{S}_{n}\right)$ shows that $S$ is an identifying code and $\gamma^{I D}\left(\bar{S}_{n}\right) \leq|S|=n$ holds. However, this bound is tight only for $n=5,6$.

Hence, in the sequel, we will consider the cases when $n \geq 7$. 
Remark 23. From the definition of $\bar{S}_{n}$, we obtain the following hyperedges of $H_{I D}\left(\bar{S}_{n}\right)$ :

(1) $N\left[s_{i}\right]=\left(C-\left\{c_{i}, c_{i-1}\right\}\right) \cup\left\{s_{i}\right\}$,

(2) $N\left[s_{i}\right] \Delta N\left[s_{j}\right]=\left\{c_{i-1}, c_{i}, c_{j-i}, c_{j}, s_{i}, s_{j}\right\}$, in particular $N\left[s_{i}\right] \Delta N\left[s_{i+1}\right]=\left\{c_{i-1}, c_{i+1}, s_{i}, s_{i+1}\right\}$,

(3) $N\left[c_{i}\right] \Delta N\left[c_{j}\right]=\left\{s_{i}, s_{i+i}, s_{j}, s_{j+1}\right\}$, in particular $N\left[c_{i}\right] \Delta N\left[c_{i+1}\right]=\left\{s_{i}, s_{i+2}\right\}$.

Theorem 24. The identifying code number of $\bar{S}_{n}$ with $n \geq 7$ is $n-1$.

Proof. Let us show that $I^{*}=\left\{c_{1}, c_{3}, s_{2}, s_{3}, s_{5}, s_{6}, \ldots, s_{n-1}\right\}$ is an identifying code. Indeed, all nodes in $C \cup S$ are separated and dominated since we have that:

$N\left[c_{i}\right] \cap I^{*}=I^{*} \backslash\left\{s_{i+1}\right\}$ with $i=1$ or $i=4$.

$N\left[c_{i}\right] \cap I^{*}=I^{*} \backslash\left\{s_{i}\right\}$ with $i=3$ or $i=n-1$.

$N\left[c_{i}\right] \cap I^{*}=I^{*} \backslash\left\{s_{i}, s_{i+1}\right\}$ with $i=2$ or $i \in\{5, \ldots, n-2\}$.

$N\left[c_{n}\right] \cap I^{*}=I^{*}$.

$N\left[s_{1}\right] \cap I^{*}=\left\{c_{3}\right\}$.

$N\left[s_{2}\right] \cap I^{*}=\left\{s_{2}, c_{3}\right\}$.

$N\left[s_{3}\right] \cap I^{*}=\left\{s_{3}, c_{1}\right\}$.

$N\left[s_{4}\right] \cap I^{*}=\left\{c_{1}\right\}$.

$N\left[s_{i}\right] \cap I^{*}=\left\{s_{i}, c_{1}, c_{3}\right\}$ with $i=5,6, \ldots, n-1$.

$N\left[s_{n}\right] \cap I^{*}=\left\{c_{1}, c_{3}\right\}$.

Also we can observe that $\left|I^{*}\right|=n-1$, hence $\gamma^{I D}\left(\bar{S}_{n}\right) \leq n-1$ follows. Let $I$ be an identifying code of $\bar{S}_{n}$, we will show that $|I| \geq n-1$.

Claim 1: There cannot be 3 consecutive nodes in $S-I$. Suppose that $s_{i} \notin I$ holds. Then we have by Remark 23(3) that $\left\{s_{i-2}, s_{i+2}\right\} \subset I$. Moreover $\left\{s_{i-1}, s_{i+1}\right\} \cap I \neq \emptyset$. $\diamond$

Claim 2: Let $S^{\prime}=\left\{s_{i_{1}}, s_{i_{2}}, \ldots, s_{i_{t}}\right\} \subset S-I$ with $t<n$ such that $s_{i_{j+1}} \geq s_{i_{j}+3}$ for all $j \in\{1, \ldots, t\}$ (the addition in the indices is modulo $n$ ). Let $H \subset I$ such that $N\left[s_{i_{j}}\right] \cap H=N\left[s_{i_{l}}\right] \cap H$ for all $j, l \in\{1, \ldots, t\}$. Then $|I| \geq|H|+t-1$.

From assumption $N\left[s_{i_{j}}\right] \cap H=N\left[s_{i_{l}}\right] \cap H$ for all $j, l \in\{1, \ldots, t\}$ then given a pair $s_{i_{j}}, s_{i_{l}}$ in $S^{\prime}$ there must be $w \in I-H$ that separates them.

According to Remark 23(2) $w \in\left\{c_{i_{j}-1}, c_{i_{j}}, c_{i_{l}-1}, c_{i_{l}}, s_{i_{j}}, s_{i_{l}}\right\}$. Moreover as $H \cap S^{\prime}=\emptyset, w \in$ $\left\{c_{i_{j}-1}, c_{i_{j}}, c_{i_{l}-1}, c_{i_{l}}\right\}$. If $w \in\left\{c_{i_{l}-1}, c_{i_{l}}\right\}$ then $w$ separates $s_{i_{l}}$ from $s_{i_{k}}$ for every $k \neq 1$. If $w \in\left\{c_{i_{j}-1}, c_{i_{j}}\right\}$ then $w$ separates $s_{i_{j}}$ from $s_{i_{k}}$ for every $k \neq j$. But $w$ does not separate the remaining pairs since $s_{i_{k+1}} \geq s_{i_{k}+3}$ for all $k \in\{1, \ldots, t\}$.

W.l.o.g. assume $w \in\left\{c_{i_{l}-1}, c_{i_{l}}\right\}$. With the same arguments used above, there is at least a node $w^{\prime} \in I-H, w^{\prime} \neq w$ that separates $s_{i_{j}}$ or $s_{i_{m}}$ from the remaining nodes in $S^{\prime}-\left\{s_{i_{l}}\right\}$. Following this idea, it is clear that there must be at least $t-1$ different nodes in $I-H$ and the lemma follows. $\diamond$

Let us partition $S-I$ into the sets $A_{i}=\left\{s_{k_{i}}\right\}$ with $i=1, \ldots, t$ and $B_{j}=\left\{s_{k_{j}}, s_{k_{j}+1}\right\}$ with $j=1, \ldots, r$. If $r=0$ then the proof follows from Claim 2. Hence, assume now that $r>0$.

Claim 3: $r=1$. Suppose to the contrary that $r \geq 2$. Let $B_{j}, B_{l}$ with $j<l$. From Claim 1 and Remark 23(3) we have that $\left\{s_{k_{j}}, s_{k_{j}+1}, s_{k_{l}}, s_{k_{l}+1}\right\} \cap I=\emptyset$ contradicting the fact that $I$ is an identifying code. $\diamond$ 
Therefore, we have that $|S \cap I|=n-t-2$. W.1.o.g. assume that $\left\{s_{1}, s_{2}\right\} \notin I$. By Remark 23(2) $\left\{c_{2}, c_{n}\right\} \cap I \neq \emptyset$. Let us assume that $c_{2} \in I$ (the same argument can be applied to $c_{n} \in I$ ).

Then $c_{2} \in N[w]$ for every $w \in\left\{s_{1}\right\} \cup\left(\bigcup_{i=1}^{t} A_{i}\right)$. Using Claim 2. with $S^{\prime}=\left\{s_{1}\right\} \cup\left(\bigcup_{i=1}^{t} A_{i}\right)$ and $H=\left\{c_{2}\right\} \cup(S \cap I)$ we obtain that $|I| \geq|H|+t=(1+n-t-2)+t=n-1$.

\section{Concluding remarks}

The identifying code problem is hard in general and challenging both from a theoretical and a computational point of view, even for special graphs like bipartite graphs [9] and split graphs [11]. Hence, a typical line of attack is to determine minimum identifying codes of special graphs (as paths [7, 14], stars [15] and cycles [7, 14]), or to provide lower and upper bounds [12, 15, 17].

In this paper, we demonstrated how polyhedral techniques can help to find identifying codes of minimum size. For that, we rely on a reformulation of the identifying code problem in terms of a set covering problem in a suitable hypergraph $H_{I D}(G)$ and study the identifying code polyhedron $P_{I D}(G)=Q^{*}\left(C_{I D}(G)\right)$ as covering polyhedron associated with its incidence matrix $C_{I D}(G)$.

We provided some general properties of the identifying code polyhedron $P_{I D}(G)$ and its canonical linear relaxation (Section 3). Afterwards, we discussed several lines to apply polyhedral techniques to the identifying code problem. In any case, the first step is to determine $H_{I D}(G)$ and its incidence matrix $C_{I D}(G)$.

If $C_{I D}(G)$ falls into a class of matrices $M$ for which the set covering polyhedron $Q^{*}(M)$ is already known, then we immediately obtain a complete description of $P_{I D}(G)$ and can deduce the exact value of $\gamma^{I D}(G)$. This turned out to be the case for stars $K_{1, n}$ (where $C_{I D}\left(K_{1, n}\right)$ equals a 2-rose $R_{n+1}^{2}$ ) and for general complete multipartite graphs $G$ (where $C_{I D}(G)$ is composed by blocks of 2-roses). Moreover, the identifying code clutter of $n$-suns $M_{n}$ turned out to equal the circulant matrix $C_{2 n}^{2}$ which implied $P_{I D}\left(M_{n}\right)=Q_{I D}\left(M_{n}\right)$. In all these cases, we obtained a complete description of $P_{I D}(G)$ and a closed formula for the exact value of $\gamma^{I D}(G)$.

A matrix $M$ is ideal if $Q^{*}(M)=Q(M)$. Hence, we can conclude from our result on $n$-suns:

Corollary 25. The identifying code clutters of $n$-suns $M_{n}$ are ideal for all $n \geq 3$.

A way to evaluate how far a nonideal matrix is from being ideal consists in classifying the inequalties that have to be added to $Q(M)$ in order to obtain $Q^{*}(M)$. In [1], a matrix $M$ is called rank-ideal if $Q^{*}(M)$ is described by rank constraints only. Thus, the results in Section 4 imply:

Corollary 26. The identifying code clutters of complete multipartite graphs $G$ are rank-ideal since rank constraints associated with cliques in $H_{I D}(G)$ suffice to describe $P_{I D}(G)$.

In general, we cannot expect identifying code clutters to be (rank-)ideal. Complete suns $S_{n}$ and their complements are examples of graphs $G$ where $C_{I D}(G)$ is far from being rank-ideal. However, an analysis of $C_{I D}(G)$ implies $\gamma^{I D}\left(S_{n}\right) \leq n$ and rised a conjecture in [3] that this bound is tight. Here, we were able to verify this conjecture by combining polyhedral and combinatorial arguments. Finally, we provided a purely combinatorial proof for $\gamma^{I D}\left(\bar{S}_{n}\right) \leq n-1$ for all complements of complete suns with $n \geq 7$.

Note that the arguments and techniques applied to complete suns are rather general and have the potential to be applied to all graphs $G$, even if their identifying code clutters are matrices with a complex structure and a complete description of $P_{I D}(G)$ involves many and complicated facets. In all such cases, an analysis of $C_{I D}(G)$ can provide, on the one hand, upper bounds for 
$\gamma^{I D}(G)$ and, on the other hand, minors of $C_{I D}(G)$ (e.g. associated with cliques or odd hypercycles in $H_{I D}(G)$ ) whose rank constraints strengthen the linear relaxation $Q_{I D}(G)$ and can be used to obtain lower bounds for $\gamma^{I D}(G)$.

Future lines of our research include to identify more facet-defining substructures in $H_{I D}(G)$ (related to minors of $C_{I D}(G)$ ) that allow us to strengthen the linear relaxation $Q_{I D}(G)$. Thereby, our goal is to obtain either the identifying code of minimum size or strong lower bounds stemming from linear relaxations of the identifying code polyhedron, enhanced by suitable cutting

planes. Recall that facets associated with deletion minors of $C_{I D}(G)$ remain facets in $P_{I D}(G)$, so according facets identified for special graphs are relevant for every graph having such subgraphs.

\section{References}

[1] G. Argiroffo, S. Bianchi: On the set covering polyhedron of circulant matrices, Discrete Optimization Vol 6-2 (2009) 162-173.

[2] G. Argiroffo, S. Bianchi, A. Wagler: Polyhedra associated with identifying codes (extended abstract), Electronic Notes in Discrete Mathematics Vol 44 (2013) 175-180.

[3] G. Argiroffo, S. Bianchi, A. Wagler: Study of identifying code polyhedra for some families of split graphs, Lecture Notes in Computer Science 8596 (2014) 13-25.

[4] G. Argiroffo, M. Carr, On the set covering polyhedron of q-roses, VI ALIO/EURO Workshop on Applied Combinatorial Optimization 2008.

[5] Auger, D.: Minimal identifying codes in trees and planar graphs with large girth, European Journal of Combinatorics 31, 1372-1384 (2010)

[6] E. Balas, S. M. Ng, On the set covering polytope: I. All the facets with coefficients in $\{0,1,2\}$, Mathematical Programming 43, (1989), pp. 57-69.

[7] T.Y. Berger-Wolf, M. Laifenfeld, A. Trachtenberg. Identifying codes and the set cover problem, Proceedings of the 44th Annual Allerton Conference on Communication, Control and Computing, Monticello, USA, 2006.

[8] Bertrand, N., Charon, I., Hudry, O., Lobstein, A.: Identifying and locating dominating codes on chains and cycles, European Journal of Combinatorics 25, 969-987 (2004)

[9] I. Charon, O. Hudry and A. Lobstein, Minimizing the size of an identifying or locating-dominating code in a graph is NP-hard. Theoretical Computer Science 290 (2003) 2109-2120.

[10] G. Cornuéjols, Combinatorial Optimization: Packing and Covering, SIAM, CBMS 74, 2001.

[11] F. Faucoud, The complexity of the identifying code problem in restricted graph classes. Combinatorial Algorithms - Proceedings of IWOCA 2013. LNCS 8288:150-163, 2013.

[12] Foucaud, F., Gravier, S., Naserasr, R., Parreau, A., Valicov, P.: Identifying codes in line graphs, Journal of Graph Theory 73, 4 (2013) 425-448

[13] D. Fulkerson, Blocking polyhedra, In: B. Haris (Ed.) Graph Theory and its Applications, Academic Press, New York (1970), pp. 93-112.

[14] J. Gimbel, B.D. Van Gorden, M. Nicolescu, C. Umstead and N. Vaiana. Location with dominating sets. Congressus Numerantium 151 (2001) 129-144.

[15] S. Gravier and J. Moncel, On graphs having a $V\{x\}$-set as an identifying code, Discrete Mathematics 307 (2007) 432-434.

[16] Gravier, S., Moncel, J., Semri, A.: Identifying codes of cycles, European Journal of Combinatorics 27, 767-776 (2006)

[17] M. G. Karpovsky, K. Chakrabarty, and L. B. Levitin, On a new class of codes for identifying nodes in graphs. IEEE Transactions on Information Theory 44 (1998) 599-611.

[18] P. Nobili, A. Sassano, Facets and lifting procedures for the set covering polytope, Mathematical Programming 45 111-137 (1989).

[19] A. Sassano, On the facial structure of the set covering polytope, Mathematical Programming 44, (1989) 181-202. 